GANIT J. Bangladesh Math. Soc. (ISSN 1606-3694) Vol. 33 (2013) 33 -39

\title{
GENERALIZED DERIVATIONS OF PRIME GAMMA RINGS
}

\author{
${ }^{1}$ Kalyan Kumar Dey, and ${ }^{2}$ Akhil Chandra Paul \\ ${ }^{1,2}$ Department of Mathematics, Rajshahi University, Rajshahi-6205, Bangladesh \\ ${ }^{1}$ kkdmath@yahoo.com, ${ }^{2}$ acpaulrubd_math@yahoo.com
}

Received 28.08.12 Accepted 31.07.13

\begin{abstract}
Let $M$ be a prime $\Gamma$-ring satisfying a certain assumption $a \alpha b \beta c=a \beta b \alpha c$ for all $a, b$, $c \in M$ and $\alpha, \beta \in \Gamma$, and let $I$ be an ideal of $M$. Assume that $(D, d)$ is a generalized derivation of $M$ and $a \in M$. If $D\left([x, a]_{\alpha}\right)=0$ or $[D(x), a]_{\alpha}=0$ for all $x \in I, \alpha \in \Gamma$, then we prove that $d(x)=p \beta[x, a]_{\alpha}$ for all $x \in I, \alpha, \beta \in \Gamma$ or $a \in Z(M)$ (the centre of $M$ ), where $p$ belongs $C(M)$ (the extended centroid of $M$ ).
\end{abstract}

Mathematics Subject Classification: 16N60, 16W25.

Keywords: Prime $\Gamma$-ring, derivation, generalized derivation, commuting mapping.

\section{Introduction}

The notion of a $\Gamma$-ring was first introduced by Nobusawa [9]. Barnes [5] weakened slightly the conditions in the definition of $\Gamma$-ring in the sense of Nabosawa [9]. Ceven and Ozturk [6] studied on Jordan generalized derivations in $\Gamma$-rings and they proved that every Jordan generalized derivation on some $\Gamma$-rings is a generalized derivation and an example of a generalized derivation and a Jordan generalized derivation for $\Gamma$-rings are given. Hvala [8] first introduced the generalized derivations in rings and obtained some remarkable results in classical rings. Generalized derivations of semiprime rings has been worked by Ali and Chaudhry [1]. They proved that $d(x)[y, z]=0$ for all $x, y, z \in R$ and the associate derivation $d$ is central. They characterized a decomposition of $R$ relative to the generalized derivations. Atteya [4] obtained some results on generalized derivations of semiprime rings. He proved that the ring $R$ contains a nonzero central ideal. Rehman [12] studied on generalized derivations acting as homomorphisms and anti-homomorphisms. He investigated the commutativity of $R$ by means if generalized derivations acting as homomorphisms and anti-homomorphisms. Aydin [3] studied on generalized derivations of prime rings. Assuming $F([x, a])=0$ or $[F(x), a]=0$ for all $x \in I$, he proved that $d(x)=$ $\lambda[x, a]$ for all $x \in I$ or $a \in Z,(F, d)$ is a generalized derivation of $R, I$ is an ideal of $R, a \in R$ and $\lambda \in C(R)$ (the extended centroid of $R$ ).

In this paper, we obtain the analogous results of Aydin [3] in $\Gamma$-rings. If $M$ is a prime $\Gamma$ ring satisfying a certain assumption (*) $a \alpha b \beta c=a \beta b \alpha c$ for all a, b, c $\in M$ and $\alpha, \beta \in \Gamma$, and $I$ is an ideal of $M$, then we prove that $d(x)=p \beta[x, a]_{\alpha}$ for all $x \in I, \alpha, \beta \in \Gamma$ or $a \in Z(M)$ (the 
centre of $M$ ), $\mathrm{p} \in C(M)$ (the extended centroid of $M$ ) by assuming that $D\left([x, a]_{\alpha}\right)=0$ or $[D(x), a]_{\alpha}=0$ for all $x \in I, \alpha \in \Gamma$, where $a \in M$.

\section{Preliminaries}

Let $M$ and $\Gamma$ be additive abelian groups. $M$ is called a $\Gamma$-ring if for all $a, b, c \in M, \alpha, \beta \in \Gamma$, the following conditions are satisfied:

(i) $a \alpha b \in M$,

(ii) $\quad(a+b) \alpha c=a \alpha c+b \alpha c, a(\alpha+\beta) b=a \alpha b+a \beta b$, $a \alpha(b+c)=a \alpha b+a \alpha c$,

(iii) $(a \alpha b) \beta c=a \alpha(b \beta c)$.

This definition of a $\Gamma$-ring is given by Barnes [5]. We represent $\mathrm{Z}(M)$ as the centre of a $\Gamma$ ring $M$. Let $M$ be a $\Gamma$-ring. A subring $I$ of $M$ is an additive subgroup which is also a $\Gamma$ ring. $A$ right ideal of $M$ is a subring $I$ such that $I \Gamma M \subset I$. Similarly a left ideal can be defined. If $I$ is both a right and a left ideal then we say that $I$ is an ideal.

The commutator $x \alpha y-y \alpha x$ will be denoted by $[x, y]_{\alpha}$. We know that $[x \beta y, z]_{\alpha}=[x, z]_{\alpha} \beta y$ $+x \beta[y, z]_{\alpha}+x[\beta, \alpha]_{z} y$

and $[x, y \beta z]_{\alpha}=y \beta[x, z]_{\alpha}+[x, y]_{\alpha} \beta z+y[\beta, \alpha]_{x} z$, for all $x, y, z \in M$ and $\alpha, \beta \in \Gamma$.

We take an assumption (*) $x \beta y \alpha z=x \alpha y \beta z$ for all $x, y, z \in M$ and $\alpha, \beta \in \Gamma$. Using the assumption the basic commutator identities reduce to

$[x \beta y, z]_{\alpha}=[x, z]_{\alpha} \beta y+x \beta[y, z]_{\alpha}$

and $[x, y \beta z]_{\alpha}=y \beta[x, z]_{\alpha}+[x, y]_{\alpha} \beta z$, for all $x, y, z \in M$ and $\alpha, \beta \in \Gamma$.

Recall that a ring $M$ is semiprime if $a \Gamma M \Gamma a=0$ implies $a=0$ and is prime if $a \Gamma M \Gamma b=0$ implies $a=0$ or $b=0$. An additive mapping $d: M \rightarrow M$ is called a derivation on $M$ if $d(x \alpha y)=d(x) \alpha y+x \alpha d(y)$ for all $x, y \in M, \alpha \in \Gamma$. An additive mapping $f: \mathrm{M} \rightarrow M$ is called commuting if $[f(x), x]_{\alpha}=0$ for all $x \in M, \alpha \in \Gamma$. It is called central if $f(x) \in Z(M)$ for all $x \in M$. Let $a \in M$, then the mapping $d: M \rightarrow M$ given by $d(x)=[a, x]_{\alpha}$ is a derivation on $M$. It is called inner derivation on $M$.

An additive mapping $D$ of $M$ into itself is called a generalized derivation of $M$, with associated derivation $d$, if there is a derivation $d$ of $M$ such that $D(x \alpha y)=D(x) \alpha y+$ $x \alpha d(y)$ for all $x, y \in M, \alpha \in \Gamma$. Obviously this notion covers the notion of a derivation (in case $D=d$ ) and a left centralizer (in case $d=0$ ). An additive mapping $D: M \rightarrow M$ is called a left centralizer if $D(x \alpha y)=D(x) \alpha y$ for all $x, y \in M, \alpha \in \Gamma$.

We refer to $[10,11]$ for the definitions of the centroid and of the extended centroid of $\Gamma$ rings.

\section{Generalized Derivations of Prime $\Gamma$-rings}

In this section, we prove our main results. Before proving our results, we need the following three lemmas which are given below. 
Lemma 3.1. Let $d$ be a derivation of a prime $\Gamma$-ring $M$ and $a$ be an element of $M$. If $a \Gamma d(x)=0$ for all $x \in M$ then either $a=0$ or $d=0$.

Proof. Let $a \in M$, and $\alpha \in \Gamma$, then $\operatorname{a\alpha d}(x)=0$. Replacing $x \beta y$ for $x,(y \in M, \beta \in \Gamma)$ we get $a \alpha d(x \beta y)=a \alpha d(x) \beta y+a \alpha x \beta d(y)=a \alpha x \beta d(y)=0$. By the primeness of $M$, we obtain either $a=0$ or $d=0$.

Lemma 3.2 Let $M$ be a $\Gamma$-ring satisfying the condition (*), $I$ be an ideal of $M$ and $(D, d)$ be a generalized derivation of $M$ and $a \in M$. If $a \notin Z(M)$ and

$[D(x), a]_{\alpha}=0$ for all $x \in I, \alpha \in \Gamma$, then $D\left([x, a]_{\alpha}\right)=0$ for all $x \in I, \alpha \in \Gamma$.

Proof. We replace $x$ by $x \delta r, r \in M, \delta \in \Gamma$, in the defining equation

$[D(x), a]_{\alpha}=0$ for all $x \in I, \alpha \in \Gamma$

and hence we obtain,

$$
\begin{aligned}
& 0=[D(x \delta r), a]_{\alpha}=[D(x) \delta r+x \delta d(r), a]_{\alpha} \\
& =[D(x) \delta r, a]_{\alpha}+[x \delta d(r), a]_{\alpha .}
\end{aligned}
$$

By using the condition $(*)$ we obtain

$[D(x) \delta r, a]_{\alpha}+[x \delta d(r), a]_{\alpha}$.

$=D(x) \delta[r, a]_{\alpha}+[D(x), a]_{\alpha} \delta r+x \delta[d(r), a]_{\alpha}+[x, a]_{\alpha} \delta d(r)$

for all $x \in I, r \in M, \alpha, \delta \in \Gamma$, which implies that

$D(x) \delta[r, a]_{\alpha}+x \delta[d(r), a]_{\alpha}+[x, a]_{\alpha} \delta d(r)=0$ for all $x \in I, r \in M, \alpha, \delta \in \Gamma$

In (2), replacing $x$ by $x \beta y,(y \in I, \beta \in \Gamma)$ and using (2), we obtain

$0=D(x \beta y) \delta[r, a]_{\alpha}+x \beta y \delta[d(r), a]_{\alpha}+x \beta[y, a]_{\alpha} \delta d(r)+[x, a]_{\alpha} \beta y \delta d(r)$

$=D(x) \beta y \delta[r, a]_{\alpha}+x \beta \mathrm{d}(\mathrm{y}) \delta[\mathrm{r}, \mathrm{a}]_{\alpha}+x \beta y \delta[d(r), a]_{\alpha}+x \beta[y, a]_{\alpha} \delta d(r)$

$+[x, a]_{\alpha} \beta y \delta d(r)$

$=D(x) \beta y \delta[r, a]_{\alpha}+x \beta d(y) \delta[r, a]_{\alpha}+x \beta\left(y \delta[d(r), a]_{\alpha}+[y, a]_{\alpha} \delta d(r)\right)$

$+[x, a]_{\alpha} \beta y \delta d(r)$

$=D(x) \beta y \delta[r, a]_{\alpha}+x \beta d(y) \delta[r, a]_{\alpha}-x \beta D(y) \delta[r, a]_{\alpha}+[x, a]_{\alpha} \beta y \delta d(r)$

$=(D(x) \beta y+x \beta d(y)-x \beta D(y)) \delta[r, a]_{\alpha}+[x, a]_{\alpha} \beta y \delta d(r)$

so we get

$(D(x) \beta y+x \beta d(y)-x \beta D(y)) \delta[r, a]_{\alpha}+[x, a]_{\alpha} \beta y \delta d(r)=0$,

for all $x, y \in I, r \in M, \alpha, \beta, \delta \in \Gamma$.

Replace $r$ by $a$ in (3), we have $[x, a]_{\alpha} \beta y \delta d(a)=0, x, y \in I, \alpha, \beta, \delta \in \Gamma$.

Since $a \notin Z(M)$ and the primeness of $I$, yields $d(a)=0$

If we substitute $s \lambda x,(s \in M, \lambda \in \Gamma)$, for $x$ in (3), then we get

$0=(D(s \lambda x) \beta y+s \lambda x \beta d(y)-s \lambda x \beta D(y)) \delta[r, a]_{\alpha}+[s \lambda x, a]_{\alpha} \beta y \delta d(r)$

$$
=((D(s) \lambda x+s \lambda d(x)) \beta y+s \lambda x \beta d(y)-s \lambda x \beta D(y)) \delta[r, a]_{\alpha}
$$




$$
\begin{gathered}
+s \lambda[x, a]_{\alpha} \beta y \delta d(r)+[s, a]_{\alpha} \lambda x \beta y \delta d(r) \\
=(D(s) \lambda x \beta y+s \lambda d(x) \beta y+s \lambda x \beta d(y)-s \lambda x \beta D(y)) \delta[r, a]_{\alpha} \\
+s \lambda[x, a]_{\alpha} \beta y \delta d(r)+[s, a]_{\alpha} \lambda x \beta y \delta d(r) \\
=D(s) \lambda x \beta y \delta[r, a]_{\alpha}+s \lambda d(x) \beta y \delta[r, a]_{\alpha}+s \lambda x \beta d(y) \delta[r, a]_{\alpha} \\
-s \lambda x \beta D(y) \delta[r, a]_{\alpha}+s \lambda[x, a]_{\alpha} \beta y \delta d(r)+[s, a]_{\alpha} \lambda x \beta y \delta d(r) \\
=(D(s) \lambda x \beta y+s \lambda d(x) \beta y) \delta[r, a]_{\alpha}+s \lambda\left((x \beta d(y)-x \beta D(y)) \delta[r, a]_{\alpha}\right. \\
\left.\quad+[x, a]_{\alpha} \beta y \delta d(r)\right)+[s, a]_{\alpha} \lambda x \beta y \delta d(r) \\
=(D(s) \lambda x \beta y+s \lambda d(x) \beta y) \delta[r, a]_{\alpha}+s \lambda\left(-D(x) \beta y \delta[r, a]_{\alpha}\right)+[s, a]_{\alpha} \lambda x \beta y \delta d(r) \\
=(D(s) \lambda x \beta y+s \lambda d(x) \beta y-s \lambda D(x) \beta y) \delta[r, a]_{\alpha}+[s, a]_{\alpha} \lambda x \beta y \delta d(r) \\
\text { and so } \quad \\
(D(s) \lambda x+s \lambda d(x)-s \lambda D(x)) \delta y \beta[r, a]_{\alpha}+[s, a]_{\alpha} \lambda x \beta y \delta d(r)=0,
\end{gathered}
$$

for all $x, y \in I, r, s \in M, \alpha, \beta, \delta, \lambda \in \Gamma$. (4)

In (4) replacing $s$ by $a$,

$(D(a) \lambda x+a \lambda d(x)-a \lambda D(x)) \beta y \delta[r, a]_{\alpha}=0$,

for all $x, y \in I, r \in M, \alpha, \beta, \delta \in \Gamma$.

Using $a \notin Z(M)$ and the primeness of $I$, we obtain

$$
D(a) \lambda x+a \lambda d(x)-a \lambda D(x)=0 .
$$

Then we have

$D(a \lambda x)=a \lambda D(x)$, for all $x \in I, \lambda \in \Gamma$,

On the other hand, since $d(a)=0$, we see that the relation

$$
D(x \lambda a)=D(x) \lambda a+x \lambda d(a)=D(x) \lambda a
$$

is reduced to $D(x \lambda a)=D(x) \lambda a$, for all $x \in I, \lambda \in \Gamma$.

$\Leftrightarrow D(x \alpha a)=D(x) \alpha a$, for all $x \in I, \alpha \in \Gamma$.

Combining (6) and (7), we arrive at

$D\left([x, a]_{\alpha}\right)=D(x \alpha a)-D(a \alpha x)=D(x) \alpha a-a \alpha D(x)=[D(x), a]_{\alpha}$ for all $x \in I, \alpha \in \Gamma$.

By using the hypothesis, we have

$$
D\left([x, a]_{\alpha}\right)=[D(x), a]_{\alpha}=0 \text {, for all } x \in I, \alpha \in \Gamma .
$$

This completes the proof.

Lemma 3.3 Let $M$ be a prime $\Gamma$ - ring satisfying the condition (*), $I$ be an ideal of $\mathrm{M},(D$, d) be a generalized derivation of $M$ and $a \in M$. If $a \notin Z(M)$ and $D\left([x, a]_{\alpha}\right)=0$ for all $x \in I$, $\alpha \in \Gamma$, then $[D(x), a]_{\alpha}=0$ for all $x \in I, \alpha \in \Gamma$.

Proof. We replace $x$ by $x \beta a(\beta \in \Gamma)$ in the defining equation $D\left([x, a]_{\alpha}\right)=0$ to obtain $0=$ $D\left([x \beta a, a]_{\alpha}\right)=D\left([x, a]_{\alpha} \beta a\right)=D\left([x, a]_{\alpha}\right) \beta a+[x, a]_{\alpha} \beta d(a)$ and so 
$[x, a]_{\alpha} \beta d(a)=0$, for all $x \in I, \alpha, \beta \in \Gamma$.

Taking $x \delta y, y \in I, \delta \in \Gamma$, instead of $x$ in (8),

$0=[x \delta y, a]_{\alpha} \beta d(a)=x \delta[y, a]_{\alpha} \beta d(a)+[x, a]_{\alpha} \delta y \beta d(a)$

and using (8) we obtain

$[x, a]_{\alpha} \delta y \beta d(a)=0$, for all $x \in I, \alpha, \beta, \delta \in \Gamma$,

By the primeness of $I$ and $a \notin Z(M)$, (9) implies that $d(a)=0$.

Now we replace $x$ by $x \lambda y,(y \in I, \lambda \in \Gamma)$ in the defining equation

$D\left([x, a]_{\alpha}\right)=0$ to obtain

$$
\begin{aligned}
0=D\left([x \lambda y, a]_{\alpha}\right)=D\left(x \lambda[y, a]_{\alpha}+[x, a]_{\alpha} \lambda y\right) \\
\quad=D\left([x, a]_{\alpha} \lambda y\right)+D\left(x \lambda[y, a]_{\alpha}\right) \\
\quad=D\left([x, a]_{\alpha}\right) \lambda y+[x, a]_{\alpha} \lambda d(y)+D(x) \lambda[y, a]_{\alpha}+x \lambda d\left([y, a]_{\alpha}\right) \\
\quad=[x, a]_{\alpha} \lambda d(y)+D(x) \lambda[y, a]_{\alpha}+x \lambda\left([d(y), a]_{\alpha}+[y, d(a)]_{\alpha}\right)
\end{aligned}
$$

Since $d(a)=0$, we have

$D(x) \lambda[y, a]_{\alpha}+[x, a]_{\alpha} \lambda d(y)+x \lambda[d(y), a]_{\alpha}=0$,

for all $x, y \in I, \alpha, \lambda \in \Gamma$,

Substitute $y \delta z,(z \in I, \delta \in \Gamma)$, instead of $y$ in equation (10) and use the equation (10), we obtain,

$$
\begin{gathered}
0=D(x) \lambda[y \delta z, a]_{\alpha}+[x, a]_{\alpha} \lambda d(y \delta z)+x \lambda[d(y \delta z), a]_{\alpha} \\
=D(x) \lambda y \delta[z, a]_{\alpha}+D(x) \lambda[y, a]_{\alpha} \delta z+[x, a]_{\alpha} \lambda d(y) \delta z \\
+[x, a]_{\alpha} \lambda y \delta d(z)+x \lambda[d(y) \delta z, a]_{\alpha}+x \lambda[y \delta d(z), a]_{\alpha} \\
=D(x) \lambda y \delta[z, a]_{\alpha}+\left(D(x) \lambda[y, a]_{\alpha}+[x, a]_{\alpha} \lambda d(y)\right) \delta z+[x, a]_{\alpha} \lambda y \delta d(z) \\
+x \lambda d(y) \delta[z, a]_{\alpha}+x \lambda[d(y), a]_{\alpha} \delta z+x \lambda y \delta[d(z), a]_{\alpha}+x \lambda[y, a]_{\alpha} \delta d(z) \\
=D(x) \lambda y \delta[z, a]_{\alpha}+\left(D(x) \lambda[y, a]_{\alpha}+[x, a]_{\alpha} \lambda d(y)+x \lambda[d(y), a]_{\alpha}\right) \delta z \\
+[x, a]_{\alpha} \lambda y \delta d(z)+x \lambda d(y) \delta[z, a]_{\alpha}+x \lambda y \delta[d(z), a]_{\alpha}+x \lambda[y, a]_{\alpha} \delta d(z) \\
=D(x) \lambda y \delta[z, a]_{\alpha}+[x, a]_{\alpha} \lambda y \delta d(z)+x \lambda d(y) \delta[z, a]_{\alpha}+x \lambda y \delta[d(z), a]_{\alpha} \\
+x \lambda[y, a]_{\alpha} \delta d(z) \\
=(D(x) \lambda y+x \lambda d(y)) \delta[z, a]_{\alpha}+[x, a]_{\alpha} \lambda y \delta d(z) \\
+x \lambda\left(y \delta[d(z), a]_{\alpha}+[y, a]_{\alpha} \delta d(z)\right) \\
=(D(x) \lambda y+x \lambda d(y)) \delta[z, a]_{\alpha}+[x, a]_{\alpha} \lambda y \delta d(z)-x \lambda D(y) \delta[z, a]_{\alpha}
\end{gathered}
$$

and so

$$
(D(x) \lambda y+x \lambda d(y)-x \lambda D(y)) \delta[z, a]_{\alpha}+[x, a]_{\alpha} \lambda y \delta d(z)=0,
$$

for all $x, y, z \in I, \alpha, \lambda, \delta \in \Gamma, \quad(11)$

Replace $x$ by $a \alpha x,(\alpha \in \Gamma)$ in equation (11), we obtain, 


$$
\begin{aligned}
& 0=(D(a \alpha x) \lambda y+a \alpha x \lambda d(y)-a \alpha x \lambda D(y)) \delta[z, a]_{\alpha}+a \alpha[x, a]_{\alpha} \lambda y \delta d(z) \\
& =D(a \alpha x) \lambda y \delta[z, a]_{\alpha}+a \alpha\left(x \lambda d(y) \delta[z, a]_{\alpha}-x \lambda D(y) \delta[z, a]_{\alpha}+[x, a]_{\alpha} \lambda y \delta d(z)\right) \\
& =D(a \alpha x) \lambda y \delta[z, a]_{\alpha}-a \alpha D(x) \lambda y \delta[z, a]_{\alpha}
\end{aligned}
$$

Hence we get

$(D(a \alpha x)-a \alpha D(x)) \lambda y \delta[z, a]_{\alpha}=0$, for all $x, y, z \in I, \alpha, \lambda, \delta \in \Gamma$.

Since $a \notin Z(M)$ and the primeness of $M$, we have

$D(a \alpha x)=a \alpha D(x)$, for all $x \in I, \alpha \in \Gamma$.

On the other hand, since $d(a)=0$,

$D(x \alpha a)=D(x) \alpha a+x \alpha d(a)=D(x) \alpha a$

Combining (13) and (14) we arrive at

$$
\begin{aligned}
& {[D(x), a]_{\alpha}=D(x) \alpha a-a \alpha D(x)} \\
& =D(x \alpha a)-D(a \alpha x)=D\left([x, a]_{\alpha}\right)=0
\end{aligned}
$$

and so

$$
[D(x), a]_{\alpha}=0, \text { for all } x \in M, \alpha \in \Gamma .
$$

Thus the proof is complete.

Theorem 3.4 Let $M$ be a $\Gamma$ - prime ring satisfying the condition (*), $I$ be an ideal of $M$, $(D, d)$ a generalized derivation of $D$ and $a \in M$. If $a \notin Z(M)$ and $D\left([x, a]_{\alpha}\right)=0$ or $[D(x), a]_{\alpha}$ $=0$ for all $x \in I, \alpha \in \Gamma$, then $d(x)=p \beta[x, a]_{\alpha}$, where $p \in C(M)$, the extended centroid of $M$, for all $x \in I, \alpha, \beta \in \Gamma$.

Proof. Since $a \notin Z(M)$ and $[D(x), a]_{\alpha}=0$ for all $x \in I, \alpha \in \Gamma$, then by Lemma 2.2 we have $D\left([x, a]_{\alpha}\right)=0$ and $d(a)=0$

By the proof of the Lemma 2.2, we have the equation (3), in the equation (3), replace $y$ by $[a, y]_{\alpha}$ then we get

$$
\begin{aligned}
0 & =\left(D(x) \beta[a, y]_{\alpha}+x \beta d\left([a, y]_{\alpha}\right)-x \beta D\left([a, y]_{\alpha}\right)\right) \delta[r, a]_{\alpha}+[x, a]_{\alpha} \beta[a, y]_{\alpha} \delta d(r) \\
& =\left(D(x) \beta[a, y]_{\alpha}+x \beta[a, d(y)]_{\alpha} \delta[r, a]_{\alpha}+[x, a]_{\alpha} \beta[a, y]_{\alpha} \delta d(r)\right. \\
& =-\left(D(x) \beta[y, a]_{\alpha}+x \beta[d(y), a]_{\alpha}\right) \delta[r, a]_{\alpha}+[x, a]_{\alpha} \beta[a, y]_{\alpha} \delta d(r)
\end{aligned}
$$

In the above equation, using the equation (10)

$$
[a, x]_{\alpha} \beta d(y)=D(x) \beta[y, a]_{\alpha}+x \beta[d(y), a]_{\alpha}
$$

in the proof of the Lemma 2.2, we obtain

$$
[a, x]_{\alpha} \beta\left(d(y) \delta[r, a]_{\alpha}-[y, a]_{\alpha} \delta d(r)\right)=0
$$

Define $h: M \rightarrow M$ by $h(x)=[a, x]_{\alpha}$, then the above equation yields

$h(x) \beta\left(d(y) \delta[r, a]_{\alpha}-[y, a]_{\alpha} \delta d(r)\right)=0$. Since $a \notin Z(M)$, by Lemma 2.2, we get $d(y) \delta[r, a]_{\alpha}=[y, a]_{\alpha} \delta d(r)$, for all $y \in I, r \in M, \alpha, \beta, \lambda \in \Gamma$. (15)

Replace $r$ by $r \lambda s,(s \in M, \lambda \in \Gamma)$, in (15) and use (15), we obtain 
$d(y) \delta r \lambda[s, a]_{\alpha}=[y, a]_{\alpha} \lambda r \delta d(s)$, for all $r, s \in M, y \in I, \alpha, \delta, \lambda \in \Gamma$,

Substitute $y \gamma z,(z \in M, \lambda \in \Gamma)$ instead of $y$ in (16) and use (16) it gives us

$d(z) \delta r \lambda[s, a]_{\alpha}=[z, a]_{\alpha} \lambda r \delta d(s)$ for all $r, s, z \in M, \alpha, \delta, \lambda \in \Gamma$,

Now, define $g: M \rightarrow M$ by $g(x)=[x, a]_{\alpha}$, then from (17) we have

$d(z) \delta r \lambda g(s)=g(z) \lambda r \delta d(s)$, for all $r, s, z \in M, \delta, \lambda \in \Gamma$.

Since $g \neq 0$, we get, for some $p \in C(M), d(x)=p \beta[x, a]_{\alpha}$, for all $x \in I, \alpha, \beta \in \Gamma$. Thus, the proof is complete.

\section{REFERENCES}

1. F. Ali and M. Anwar Chaudhry, On generalized derivations of semiprime rings, International J of Algebra, 4, 14, (2010), 677 - 684.

2. F. Ali and M. Anwar Chaudhry, Dependent elements of derivations on semiprime rings, International Journal of Mathematics and Mathematical Sciences, 2009.

3. N. Aydin, A note on generalized derivations of prime rings, International J. of Algebra, 5, $1,(2001), 17-23$.

4. M. Jabel Atteya, On generalized derivations of Semiprime rings, International Mathematical Journal of Algebra, 4, 12, (2010), 591-598.

5. W. E. Barnes, On the $\Gamma$-rings of Nabusawa, Pacific J. Math., 18, (1966), 411-422.

6. Y. Ceven and M. Ali Ozturk, On Jordan generalized derivations in Gamma rings, Hacettepe Journal of Mathematics and Statistics, 33, (2004), 11-14.

7. Kalyan Kumar Dey, Akhil Chandra Paul and Isamiddin S. Rakhimov, Generalized derivations in semiprime gamma-rings, International Journal of Math. and Math. Sci., 2012, doi:10.1155/2012/270132

8. B. Hvala, Generalized derivations in rings, Comm. Algebra, 26, 4, (1998), 1147-1166.

9. N. Nabusawa, On a generalization of the Ring Theory, Osaka J. Math., 1, (1964), 65-75.

10. M. A. Ozturk and Y. B. Jun, On the centroid of the prime $\Gamma$-ring, Comn. Korean Math. Soc., 15(3), (2000), 469-477.

11. M. A. Ozturk and Y. B. Jun, On the centroid of the prime $\Gamma$-ring II, Turk. J. Math., 25, (2001), 367-377.

12. N. Rehman, On commutativity of rings with generalized derivations, Math. J. Okayama Univ., 44, (2002), 43-49. 